\title{
Percepción de satisfacción entre beneficiarios del programa de alimentación escolar en Bucaramanga-Colombia
}

\author{
Perception of satisfaction among beneficiaries of the \\ school food program of Bucaramanga-Colombia
}

\author{
Lucila Niño-Bautista $^{1}$; Edna-Magaly Gamboa-Delgado ${ }^{1}$; Tatiana Serrano-Mantilla ${ }^{1}$
}

Forma de citar: Niño-Bautista L, Gamboa-Delgado EM, Serrano-Mantilla T. Percepción de satisfacción entre beneficiarios del programa de alimentación escolar en Bucaramanga-Colombia. Rev Univ Ind Santander Salud. 2019; 51(3): 240-250. doi: http://dx.doi.org/10.18273/revsal.v51n3-2019007 (c) (1)

\section{Resumen}

Introducción: El Programa de Alimentación Escolar PAE en Colombia es uno de los más antiguos orientado a la protección de la población infantil para potenciar su desarrollo y disminuir la deserción escolar; sin embargo, no hay investigación suficiente que permita evidenciar avances y logros del programa. Objetivo: Determinar la prevalencia en la percepción de satisfacción de los beneficiarios del Programa de Alimentación Escolar - PAE en Bucaramanga, Colombia y sus factores asociados. Metodología: Se realizó en 2017 un estudio cuantitativo de corte transversal analítico. La población correspondió al universo de escolares beneficiarios del PAE en la ciudad $(\mathrm{N}=31.230)$ y la muestra a 401 escolares la cual se dividió en dos grupos según edad: grupo 1 (de 5 a 8 años) y grupo 2 (de 9 a 17 años). Las encuestas para evaluar los componentes sensorial, organizacional y social, fueron sometidas a prueba piloto para validación aparente o facial y ajustadas según resultados. Una vez recogidos los datos se hizo análisis descriptivo a características de interés; para corroborar diferencias estadísticamente significativas se usó la prueba de $\operatorname{chi}^{2}$ y T de Student y se realizaron análisis bivariados y multivariados entre las características de interés y la percepción de satisfacción usando regresión lineal simple y múltiple. Resultados: Los estudiantes mostraron un nivel alto de percepción de satisfacción frente a las raciones recibidas, siendo los más satisfechos los de nivel socioeconómico bajo y medio bajo (Coef $B$ 2,43; IC 95\%: 1,61-3,25) y los que recibieron ración preparada en sitio (niños de 5 a 8 años), (Coef $B$ 8,36; IC 95\%: 7,08-9,64). Se encontró que el 25\% de los estudiantes de nivel socioeconómico medio-medio y medio-alto botaba los alimentos a la basura. Conclusiones: Estos hallazgos mostraron la necesidad prioritaria de ser más rigurosos en la focalización de los beneficiarios del programa, así como llevar a cabo otros ajustes planteados en el estudio, para incrementar la calidad del programa PAE de Bucaramanga.

Palabras clave: Alimentación escolar; Satisfacción personal; Percepción; Asistencia alimentaria; Programas de alimentación; Satisfacción con la alimentación; Almuerzo escolar; Hábitos alimentarios; Consumo de alimentos; Programa de alimentación escolar.

1. Universidad Industrial de Santander, Bucaramanga, Colombia.

Correspondencia: Lucila Niño Bautista. Dirección: Carrera 32 29-31 Instituto PROINAPSA, Bucaramanga. Teléfono: +577 6450006. Correo electrónico: lucinino@hotmail.com 


\begin{abstract}
Introduction: The PAE School Feeding Program in Colombia is one of the oldest oriented to the protection of the child population to enhance their development and decrease school dropouts; however, there is not enough research to show progress and achievements of the program. Objective: To determine the prevalence of perception of satisfaction of the beneficiaries of the School Feeding Program, in Bucaramanga, Colombia and its associated factors. Methodology: An analytical cross-sectional study was carried out in 2017 with an empirical nonexperimental analytical approach. The population corresponded to the universe of PAE schoolchildren in the city $(\mathrm{N}=31,230)$ and the sample to 401 students which was divided into two groups according to age: group 1 (from 5 to 8 years) and group 2 (from 9 to 17 years). The surveys to evaluate the sensory, organizational and social components were submitted to a pilot test for apparent or facial validation and adjusted according to results. Once the data was collected, a descriptive analysis was made to the characteristics of interest; To corroborate statistically significant differences, the chi ${ }^{2}$ and Student's T test were used and bivariate and multivariate analyzes were performed between the characteristics of interest and the perception of satisfaction using simple and multiple linear regression. Results: The students showed a high level of satisfaction perception compared to the rations received, being the most satisfied those low and medium low socioeconomic status (Coef $B$ 2.43; 95\%CI: 1.61-3.25) and those who received ration prepared on site (children 5 to 8 years old), (Coef $B$ 8.36; 95\%CI: 7.08-9.64). Conclusions: These findings showed the priority need to be more rigorous in the targeting of the beneficiaries of the program, as well as carry out other adjustments proposed in the study, to increase the quality of the PAE program in Bucaramanga.
\end{abstract}

Keywords: School feeding; Personal satisfaction; Perception; Food assistance; Alimentary programs; Satisfaction with food-related life; School lunch; Eating habits; Food Consumption; School food programs.

\section{Introducción}

Los programas de alimentación que se desarrollan en el contexto escolar en el mundo tienen como finalidad principal la protección social dirigida a potenciar el desarrollo de niños, adolescentes y la prevención de la deserción escolar así mismo, apoyan objetivos de educación y de salud, favorecen el desarrollo del capital humano y promueven hábitos alimentarios adecuados ${ }^{1-3}$.

En América Latina y el Caribe, según encuesta realizada en 2016 por el Programa Mundial de Alimentos (PMA), el programa de alimentación escolar - PAE- tuvo una participación de 73.702 .225 beneficiarios en 16 países, representando una oportunidad de contribuir al logro del Objetivo 2 de Desarrollo Sostenible de la Agenda 2030, conocido como "Hambre Cero"; la inversión anual estimada fue de 4 mil millones de dólares. La mayor cobertura de participación la tuvieron Brasil con 41.500.000, México con 6.357.712 y Colombia con 4.029.869 beneficiarios. Es importante destacar que estos programas presentan una alta variabilidad en cuanto a costos, cobertura, calidad y sostenibilidad en los diferentes países ${ }^{4}$.

Respecto al estudio de la percepción de satisfacción frente a la alimentación en el ámbito escolar, ésta se relaciona con los modelos de índices de satisfacción del cliente. En general, tienen en cuenta dos referentes primarios de la satisfacción: la percepción del cliente frente al servicio o producto y la expectativa frente al desempeño del servicio 5 . La satisfacción frente a la alimentación también puede ser influenciada por factores como preferencia hacia algunos alimentos, comportamientos saludables, edad, composición del núcleo familiar, género, y nivel socioeconómico de las personas ${ }^{6}$, así como el servicio que prestan las personas que atienden al beneficiario ${ }^{7}$.

El programa PAE en Colombia, se ha propuesto i) contribuir al acceso y la permanencia escolar de los niños y adolescentes registrados en la matrícula oficial, ii) mejorar su capacidad de aprendizaje mediante el suministro de un complemento alimentario que aporta calorías y nutrientes durante el calendario escolar y iii) fomentar estilos de vida saludables. La focalización del programa se realiza bajo el enfoque de protección integral y es dirigida a la población económica y socialmente más vulnerable ${ }^{8}$. A partir del año 2017 el Programa Mundial de Alimentos PMA, reenfocó su apoyo a las prioridades del Gobierno Nacional en materia de consolidación de la paz con el objetivo de aportar a las soluciones sostenibles para las víctimas del conflicto9. Es así como el PAE, al incluir objetivos de diversos sectores, ha resaltado la necesidad de articulación intersectorial e interinstitucional para lograr el máximo impacto sobre la educación y el desarrollo humano ${ }^{10}$. 
Sobre la percepción de satisfacción de estudiantes con el PAE en Colombia, sólo se identificó un estudio realizado en el departamento de Vaupés donde participaron 849 escolares de 16 sedes educativas, en el cual el $65 \%$ se expresó satisfecho, el $27 \%$ insatisfecho y el restante $8 \%$ no respondió ${ }^{11}$.

En el contexto Latinoamericano, un estudio chileno sobre la evaluación del programa PAE en el país, encontró que el nivel de satisfacción global en los escolares de educación básica fue del $66 \%$ y en los estudiantes de educación media de 72,3\%. Al desagregar el indicador por las dimensiones de análisis, la satisfacción frente a los alimentos recibidos fue del $54 \%$ y el $52 \%$ en los dos grupos mencionados y la evaluación de la importancia del PAE fue de $66 \%$ y $72,3 \%$ respectivamente ${ }^{12}$.

En otro estudio descriptivo en el área urbana de la ciudad de Santa Fe - Argentina, que buscó identificar las percepciones vinculadas a los componentes del programa de recuperación del niño en riesgo nutricional, el 99\% de los encuestados reportó alto nivel de satisfacción, mejoramiento de conocimientos y prácticas alimentarias, diversificación de la alimentación cotidiana y fortalecimiento del vínculo madre-hijo ${ }^{13}$.

En contraste, en Asunción - Paraguay se llevó a cabo un estudio para evaluar la aceptación y adecuación a requerimientos nutricionales del almuerzo en 102 escolares de dos escuelas públicas. Se encontró que sólo a tres de 10 niños les agradaban las comidas que les sirvieron y la adecuación de energía y proteínas no se ajustó a los requerimientos recomendados ${ }^{14}$.

Entre tanto en Corea del Sur, se evaluó la satisfacción con el servicio de comida escolar en una escuela intermedia con 680 estudiantes, encontrando: satisfacción con el ambiente para comer y agrado con la cantidad, diversidad de tipos de sopa, postre y el costo de la comida escolar en la cafetería; el grupo de niñas mostró una satisfacción significativamente mayor que el grupo de niños ${ }^{15}$. De manera similar, en Colorado (EEUU) se realizó un estudio en tres escuelas para determinar la satisfacción de los estudiantes de secundaria con la experiencia de almuerzo. Los estudiantes percibieron que aspectos como el atractivo visual, el aroma y el sabor requerían ser mejorados en los alimentos servidos ${ }^{16}$.

En general las investigaciones anteriormente presentadas sobre la satisfacción de los estudiantes con los alimentos recibidos en la institución educativa, fueron de carácter descriptivo y la satisfacción global estuvo entre el 30 y $75 \%$. En los estudios que analizaron variables independientes, el sexo y el nivel de escolaridad tuvieron relación con la satisfacción por los alimentos recibidos.

Conocer la percepción de satisfacción que tienen los beneficiarios en un programa de alimentación cobra relevancia en tanto que permite detectar puntos clave para hacer ajustes y lograr mejores resultados. No obstante, medir la percepción de satisfacción de los beneficiarios del PAE representó un reto de especial importancia y complejidad en razón al tema objeto de estudio ${ }^{17}$, el no haber hallado instrumentos validados y la escases extrema de estudios sobre el tema en Colombia y la región. Ante la necesidad de aportar resultados de investigación que permitan mejorar la calidad de los programas de alimentación escolar, el objetivo de este estudio fue determinar la prevalencia de percepción de satisfacción de los beneficiarios del Programa de Alimentación Escolar - PAE en Bucaramanga, Colombia y sus factores asociados.

\section{Metodología}

Se realizó un estudio de corte transversal. La población de este estudio correspondió al universo de escolares del sector público beneficiarios del Programa PAE de Bucaramanga en $2017(\mathrm{~N}=31.230)$. Se calculó el tamaño de muestra teniendo en cuenta: población de beneficiarios, prevalencia de percepción de satisfacción de $50 \%$, nivel de confianza de $95 \%$ y efecto de diseño de 1.

La muestra, conformada por 401 escolares, fue clasificada en dos grupos considerando el nivel de desarrollo de la lecto-escritura para diligenciamiento de la encuesta y la proporcionalidad de su representación en la población así: Grupo 1, escolares de 5 a 8 años de edad y Grupo 2, estudiantes de 9 a 17 años de edad. Así mismo se seleccionaron, mediante muestreo aleatorio simple, 18 instituciones educativas en las comunas de la ciudad de tal manera que representaran los niveles socio económicos donde se encuentran los beneficiarios del programa; así se logró equilibrio de instituciones por territorio al aplicar las encuestas. Al interior de las instituciones educativas, el grupo de estudiantes para el estudio fue seleccionado mediante muestreo aleatorio simple y posterior a ese proceso, se verificó el cumplimiento de los criterios de elegibilidad hasta completar la muestra: i) estudiante activo en las instituciones educativas seleccionadas, ii) confirmación de pertenencia al programa PAE y iii) autorización o aceptación de participar en el estudio. 
La variable dependiente del estudio fue la percepción de satisfacción por parte de los beneficiarios del programa. El grupo investigador consideró la satisfacción como la confluencia entre las expectativas respecto al servicio y producto que reciben, con la percepción al obtener los servicios y productos del programa ${ }^{4,16}$. Con base en esta definición se establecieron tres componentes a evaluar: componente sensorial de los alimentos recibidos (sabor, color, olor, temperatura, y textura de los alimentos), componente organizacional (hora de servido del refrigerio y almuerzo, diversidad de los alimentos, comodidad en el sitio donde toma los alimentos, orden y respeto del turno en las filas, tranquilidad para comer, limpieza, ventilación y orden del lugar donde reciben los alimentos) y componente social (amabilidad del personal que ofrece los servicios en el programa, percepción de ambiente agradable para comer, poder conversar con los compañeros, importancia de recibir los alimentos, presión para comer y conductas como llevarse los alimentos para su casa, regalar los alimentos a compañeros, botar los alimentos a la basura).

Para codificar los puntajes de percepción de satisfacción de cada componente se le asignó un punto a cada respuesta afirmativa que correspondiera a aspectos positivos relacionados con la satisfacción. De esta manera la variable dependiente tomó un valor numérico entre 1 y 27 puntos.

Además, se analizaron variables independientes como edad, sexo, tipo de ración recibida, nivel socio económico de la zona donde está localizada la institución educativa y número de personas que compartían la alimentación en la familia.

Las encuestas fueron elaboradas incluyendo seis ítems de orden socio demográfico y entre 20 y 24 ítems (según grupo de estudiantes) relacionados con la percepción de satisfacción, con adecuaciones en el lenguaje dependiendo del grupo etáreo de estudiantes al que iba dirigida. La opción de respuesta para cada ítem fue dicotómica (si - no).

Para la comprensión y pertinencia de las preguntas, teniendo en cuenta las características del programa, se diseñaron cuatro formatos de encuestas diferentes así; (1) para escolares de 5 a 8 años de edad que recibieran ración industrializada de, aproximadamente, 418 Kilocalorías, (refrigerio entregado en el descanso de la jornada escolar);(2) para escolares de esa misma edad que recibieran ración preparada en sitio (porción alimentaria de 541 Kilocalorías complemento del almuerzo que consumirían los escolares al llegar al hogar); (3) para estudiantes de 9 a 17 años de edad que recibieran ración industrializada de 495 Kilocalorías, aproximadamente; (4) para estudiantes de esa misma edad que recibieran ración preparada en sitio de 735 kilocalorías.

Las encuestas fueron sometidas a una prueba piloto para validación aparente o facial, con dos grupos de escolares de 5 a 8 años y de 9 a17 años de instituciones distintas a las de la muestra de análisis. Con base en sus resultados se hicieron ajustes en las encuestas antes de su utilización en el estudio.

Previo al diligenciamiento de las encuestas se envió al cuidador principal de los escolares de 5 a 7 años un mensaje con información básica sobre el estudio, utilización de resultados y manejo ético de la información recolectada; su firma al final del documento acreditó el asentimiento informado para la participación del escolar. Con los estudiantes mayores se dio la información pertinente de manera presencial y se hizo claridad sobre su participación voluntaria. A quienes optaron por no participar se les respetó su decisión.

El diligenciamiento de las encuestas fue posterior a la capacitación y estandarización del equipo encuestador, conformado por nutricionistas dietistas e ingenieros de alimentos. Los encuestadores fueron distribuidos en las 18 instituciones educativas seleccionadas en zonas urbanas y rurales del municipio. A los escolares de 5 a 8 años - Grupo 1, se les leyeron los ítems de la encuesta dándoles explicaciones y ejemplos cuando tuvieron alguna dificultad de comprensión. Los estudiantes de 9 a 17 años - Grupo 2, auto diligenciaron la encuesta en su salón de clases luego de recibir las orientaciones para su llenado y al finalizar la entregaron al encuestador responsable del salón.

Concluido el diligenciamiento de las encuestas se elaboró la base de datos utilizando el protocolo para garantizar su calidad: doble digitación en Excel por diferente persona, corrección de errores detectados y reimpresión de base de datos definitiva la cual se transfirió al paquete estadístico Stata $\mathrm{v}$ 12.0, para realizar los análisis y generar resultados.

\section{Análisis Estadístico}

Se realizó análisis descriptivo de las características de interés de acuerdo al objetivo del estudio en el cual las variables continuas se presentaron como medias con su respectiva Desviación Estándar (D.E) 
y las variables categóricas como proporciones. Para evaluar si existían diferencias estadísticamente significativas entre variables categóricas se usó la prueba $\mathrm{Chi}^{2}$ y entre las variables continuas la prueba $\mathrm{T}$ de Student. Se realizaron análisis para evaluar las diferencias en cuanto a las características sociodemográficas de los participantes según el sexo y a la percepción de satisfacción de los beneficiarios del programa, según el grupo etáreo y tipo de ración recibida. Se realizaron análisis bivariados y multivariados, previo análisis del cumplimiento de supuestos, entre las características de interés y la percepción de satisfacción usando modelos de regresión lineal simple y múltiple.

\section{Resultados}

\section{Características Sociodemográficas}

Se analizó muestra representativa de 401 escolares beneficiarios del PAE de Bucaramanga - Colombia. La edad promedio de los escolares del grupo 1 fue 6,1 años y de los estudiantes del grupo 2, de 12,05 años. El $53,6 \%$ de la población fue de sexo femenino.

Tabla 1. Características generales de estudiantes beneficiarios del Programa de Alimentación Escolar según el sexo. Bucaramanga, Colombia 2017.

\begin{tabular}{|c|c|c|c|c|}
\hline $\begin{array}{c}\text { Características } \\
\text { sociodemográficas }\end{array}$ & $\begin{array}{c}\text { Todos (n=401) } \\
\text { n (\%) ó Media [D.E] }\end{array}$ & $\begin{array}{c}\text { Femenino }(\mathrm{n}=215) \\
\text { n }(\%) \text { ó Media [ D.E] }\end{array}$ & $\begin{array}{l}\text { Masculino (n=186) } \\
\text { n (\%) ó Media [ D.E] }\end{array}$ & Valor $\mathrm{p}$ \\
\hline \multicolumn{5}{|l|}{ Edad } \\
\hline Grupo 1 (5 a 8 años) & $6.14[1.19]$ & $6.28[1.18]$ & $6.0[1.20]$ & $0.125 f$ \\
\hline Grupo 2 (9 a 17 años) & $12.05[2.36]$ & $12.65[2.31]$ & $12.05[2.38]$ & $0.028 f$ \\
\hline \multicolumn{5}{|l|}{ Nivel socioeconómico } \\
\hline Medio y Medio Alto & $101(25.19)$ & $45(20.93)$ & $56(30.11)$ & $0.035 \dagger$ \\
\hline Bajo y Medio Bajo & $300(74.81)$ & $170(79.07)$ & $130(69.89)$ & \\
\hline \multicolumn{5}{|l|}{$\begin{array}{l}\text { Número de estudiantes según } \\
\text { personas que comparten la } \\
\text { alimentación en la familia }\end{array}$} \\
\hline 1 a 4 personas & $265(65.09)$ & $132(61.40)$ & $129(69.35)$ & $0.095 \dagger$ \\
\hline 5 o más personas & $140(34.91)$ & $83(38.60)$ & $57(30.65)$ & \\
\hline \multicolumn{5}{|l|}{$\begin{array}{l}\text { Grupo etáreo por tipo de ración } \\
\text { alimentaria }\end{array}$} \\
\hline Grupo 2, ración industrializada & $124(30.92)$ & $77(35.81)$ & $47(25.27)$ & $0.115 \dagger$ \\
\hline Grupo 2, ración preparada en sitio & $157(39.15)$ & $80(37.21)$ & $77(41.40)$ & \\
\hline Grupo 1, ración industrializada & $62(15.46)$ & $32(14.88)$ & $30(16.13)$ & \\
\hline Grupo 1 , ración preparada en sitio & $58(14.46)$ & $26(12.09)$ & $32(17.20)$ & \\
\hline
\end{tabular}

D.E: Desviación Estándar; $£$ : Valor p mediante prueba T de Student; †: Valor p mediante prueba $\mathrm{x}^{2}$.

Fuente: elaboración propia.

El $75 \%$ de los escolares participantes estudiaban en instituciones educativas ubicadas en áreas de nivel socio económico bajo $(29,6 \%$ nivel bajo-bajo y $45,1 \%$ nivel medio-bajo), y el resto en nivel socio económico medio (17,4\% nivel medio-medio y $7,7 \%$ nivel medio-alto).

En cuanto al tamaño de la familia (número de personas que compartían la alimentación en el hogar), más de la mitad de los hogares estaban por encima de cuatro integrantes $(41,7 \%$ por uno a tres; $49,1 \%$ cuatro a seis y $9,2 \%$ por siete y más integrantes).
Prácticas utilizadas por quienes no consumían los alimentos ofrecidos por el programa

Compartir refrigerio con la familia: en el grupo 2 una cuarta parte de estudiantes $(24,4 \%)$ guardó el refrigerio o ración industrializada para compartirlo con su familia; en el grupo 1, solo el 12,7\% la llevó al hogar. Regalar el refrigerio o el almuerzo a un compañero de clase: esta práctica también fue más utilizada por los estudiantes del grupo $2(33,2 \%)$. Botar el refrigerio a la basura: en esta práctica, el 5,7\% de los integrantes del grupo 1 la utilizaron vs el $0,7 \%$ del grupo 2 . 
La práctica de llevar refrigerio o ración industrializada al hogar no estuvo relacionada estadísticamente con el número de miembros de la misma $(\mathrm{p}=0,578)$ y respecto al nivel socio económico $(\mathrm{p}=0,062)$; estas diferencias podrían ser aceptadas al $10 \%$ de significancia, lo cual sugiere la posibilidad de que con un tamaño de muestra mayor se pudiera encontrar esa relación de manera más evidente. Se halló relación estadísticamente significativa entre regalar la comida a los compañeros y pertenecer a los niveles socio económicos bajos $(\mathrm{p}=0,000)$, así como entre botar a la basura el refrigerio o el almuerzo y ser parte de los niveles socio económicos medios $(\mathrm{p}=0,000)$.
Percepción de Satisfacción de los beneficiarios del programa

La Tabla 2 presenta las frecuencias de respuestas positivas en los tres componentes de la satisfacción (sensorial, organizacional y social), en los cuales sí hubo diferencias significativas en los grupos 1 y 2 por tipo de ración recibida. En general, los resultados muestran que los niños y niñas del grupo 1 tuvieron una percepción de satisfacción más alta en la mayoría de aspectos evaluados.

Tabla 2. Percepción de Satisfacción de los beneficiarios del Programa de Alimentación Escolar, según componentes sensorial, organizacional y social ( $\%$ de respuestas positivas).

\begin{tabular}{|c|c|c|c|c|c|}
\hline \multirow[b]{2}{*}{$\begin{array}{l}\text { Componentes e } \\
\text { ítems en la encuesta }\end{array}$} & \multicolumn{2}{|c|}{$\begin{array}{l}\text { Grupo } 1 \\
5 \text { a } 8 \text { años }\end{array}$} & \multicolumn{2}{|c|}{$\begin{array}{c}\text { Grupo } 2 \\
9 \text { a } 17 \text { años }\end{array}$} & \multirow[b]{2}{*}{ Valor $\mathbf{p}$} \\
\hline & $\begin{array}{c}\text { Ración } \\
\text { industrializada } \\
(n=62)\end{array}$ & $\begin{array}{c}\text { Ración preparada } \\
\text { en sitio } \\
(\mathrm{n}=\mathbf{5 8})\end{array}$ & $\begin{array}{c}\text { Ración } \\
\text { industrializada } \\
(\mathrm{n}=124)\end{array}$ & $\begin{array}{l}\text { Ración } \\
\text { preparada en sitio } \\
(n=157)\end{array}$ & \\
\hline & $\mathrm{n}(\%)$ & $\mathrm{n}(\%)$ & $\mathrm{n}(\%)$ & $\mathrm{n}(\%)$ & \\
\hline \multicolumn{6}{|c|}{ Componente Sensorial } \\
\hline $\begin{array}{l}\text { Le gusta el sabor de los alimentos } \\
\text { que le dan en el Refrigerio/ } \\
\text { Almuerzo }\end{array}$ & $60(96.77)$ & $56(96.55)$ & $101(81.45)$ & $124(80.52)$ & 0.001 \\
\hline $\begin{array}{l}\text { Al oler los alimentos le dan ganas } \\
\text { de comerlos }\end{array}$ & $61(98.39)$ & $56(96.55)$ & $100(80.65)$ & $138(87.90)$ & 0.001 \\
\hline $\begin{array}{l}\text { Si el R/A* tiene varios colores le } \\
\text { dan más ganas de comerlo }\end{array}$ & $50(80.65)$ & $49(84.48)$ & $65(52.42)$ & $93(59.24)$ & 0.000 \\
\hline \multicolumn{6}{|c|}{ Componente Organizacional } \\
\hline $\begin{array}{l}\text { Al recibir el } \mathrm{R} / \mathrm{A} \text { hay orden en las } \\
\text { filas }\end{array}$ & $53(85.48)$ & 45 (77.59) & $63(50.81)$ & $86(54.78)$ & 0.000 \\
\hline Se respeta el turno en la fila & $9(100.00)$ & 37 (63.79) & $52(41.94)$ & $91(57.96)$ & 0.000 \\
\hline $\begin{array}{l}\text { Le alcanza el tiempo para comer } \\
\text { tranquilo el R/A }\end{array}$ & $58(93.55)$ & $49(84.48)$ & $71(57.26)$ & $116(73.89)$ & 0.000 \\
\hline \multicolumn{6}{|c|}{ Componente Social } \\
\hline $\begin{array}{l}\text { A la hora del R/A puede conversar } \\
\text { con sus compañeros }\end{array}$ & $35(56.45)$ & $40(68.97)$ & $104(83.87)$ & $143(91.08)$ & 0.000 \\
\hline $\begin{array}{l}\text { Le parece importante recibir el R/A } \\
\text { del Colegio }\end{array}$ & $62(100.00)$ & $56(96.55)$ & $120(97.56)$ & 39 (31.97) & 0.000 \\
\hline
\end{tabular}

$* \mathrm{R} / \mathrm{A}$ : refrigerio o almuerzo

Fuente: elaboración propia.

Focalizando los componentes analizados, el sensorial tuvo porcentajes más altos de satisfacción en ambos grupos y el componente organizacional los más bajos; en el componente social se encontró manejo diferencial en poder conversar con los compañeros durante el refrigerio y almuerzo: en el grupo 1 no se podía conversar en tanto que el grupo 2 sí.
Solo se encontró diferencia significativa $(\mathrm{p}=0,003)$ de la satisfacción de los estudiantes según el nivel socio económico (media de puntaje de satisfacción en niveles socio económicos bajo-bajo y medio-bajo: 17,8, D.E 4,68 vs media de puntaje de satisfacción en niveles medio-medio y medio-alto; 14,1 , D.E 3,74 ; $\mathrm{p}=0.001$ ). 


\section{Percepción de satisfacción de acuerdo con el tipo de ración alimentaria recibida}

$\mathrm{Al}$ comparar puntajes de satisfacción por componente y tipo de ración alimentaria por grupo etáreo, los menores puntajes correspondieron a componentes sensorial y organizacional del grupo 2 en ración industrializada; y el grupo 1 que recibió ración industrializada mostró el menor puntaje para el componente social (ver Tabla 3).

Tabla 3. Percepción de satisfacción por componentes (sensorial, organizacional y social) según el tipo de ración recibida por los beneficiarios del Programa de Alimentación Escolar.

\begin{tabular}{|c|c|c|c|c|c|c|}
\hline \multirow{3}{*}{ Tipo de ración recibida } & \multicolumn{6}{|c|}{ Componente de la Percepción de Satisfacción } \\
\hline & \multicolumn{2}{|c|}{ Sensorial } & \multicolumn{2}{|c|}{ Organizacional } & \multicolumn{2}{|c|}{ Social } \\
\hline & Media (D.E) & $\mathbf{p}$ & Media (D.E) & $\mathbf{p}$ & Media (D.E) & $\mathbf{p}$ \\
\hline & & 0,000 & & 0,002 & & 0,001 \\
\hline Grupo 2: Ración industrializada & $4,14(1,26)$ & & $3,96(1,41)$ & & $5,34(0,94)$ & \\
\hline Grupo 2: Ración preparada en sitio & $4,6(1,49)$ & & $7,52(2,89)$ & & $7,38(1,41)$ & \\
\hline Grupo 1: Ración industrializada & $4,4(0,77)$ & & $7,61(0,81)$ & & $5,16(1,10)$ & \\
\hline Grupo 1: Ración preparada en sitio & $5,98(1,16)$ & & $9,39(1,64)$ & & $4,79(0,89)$ & \\
\hline
\end{tabular}

D.E: Desviación Estándar

Fuente: elaboración propia.

Factores Asociados a la percepción de satisfacción en escolares beneficiarios del PAE

Los análisis múltiples, posteriores al ajuste por sexo y edad, mostraron que el tipo de ración recibida según el grupo etáreo y el nivel socio económico, se asociaron de manera significativa con el puntaje de percepción de satisfacción frente al Programa. Al tomar como categoría de referencia al grupo 2 que recibió ración industrializada, se encontró que el puntaje total de percepción de satisfacción fue mayor en escolares del grupo 1 que recibieron ración preparada en sitio, seguido por quienes recibieron ración industrializada y finalmente por los estudiantes del grupo 2 que recibieron ración preparada en sitio. Con relación al nivel socio económico, los estudiantes que pertenecían a niveles bajos presentaron mayores puntajes de percepción de satisfacción (ver Tabla 4). Aunque no se encontraron diferencias significativas referidas a considerar importante recibir el refrigerio o el almuerzo ofrecido por el PAE, cabe resaltar que la mayoría de los participantes del estudio (68\%) manifestó que sí era importante recibirlo, siendo menor esta percepción en los estudiantes del grupo 2 que recibían ración preparada en sitio $(32 \%)$.

Tabla 4. Factores asociados a la percepción de satisfacción en estudiantes beneficiarios del Programa de Alimentación Escolar.

\begin{tabular}{|c|c|c|c|c|c|c|}
\hline \multirow{2}{*}{ Factores de Riesgo } & \multicolumn{3}{|c|}{ Modelos Crudos } & \multicolumn{3}{|c|}{ Modelos ajustados por sexo y edad } \\
\hline & Coef B & IC $95 \%$ & $\mathbf{p}$ & Coef B & IC $95 \%$ & $\mathbf{p}$ \\
\hline $\begin{array}{l}\text { Sexo } \\
\text { Femenino }\end{array}$ & REF & & REF & & REF & \\
\hline Masculino & $-0,36$ & $-1,25-0,52$ & 0,419 & $-0,63$ & $-1,32-0,04$ & 0,068 \\
\hline Edad & 0,11 & $-0,01-0,23$ & 0,075 & 0,34 & $0,16-0,51$ & 0,000 \\
\hline \multicolumn{7}{|c|}{ Tipo de ración recibida, según grupo de edad } \\
\hline Grupo 2: Ración industrializada & REF & & REF & & REF & \\
\hline Grupo 2: Ración preparada en sitio & 6,06 & $5,21-6,90$ & 0,000 & 5.44 & $4,54-6,35$ & 0,000 \\
\hline Grupo 1: Ración industrializada & 3,71 & $2,62-4,81$ & 0,000 & 6,32 & $4,87-7,77$ & 0,000 \\
\hline Grupo 1, Ración preparada en sitio & 6,71 & $5,59-7,83$ & 0,000 & 8,36 & $7,08-9,64$ & 0,000 \\
\hline \multicolumn{7}{|l|}{ Nivel socioeconómico } \\
\hline Nivel Medio-medio y Medio alto & REF & & REF & & REF & \\
\hline Nivel Bajo-bajo y nivel medio-bajo & 1,67 & $0,66-2,68$ & 0,001 & 2,43 & $1,61-3,25$ & 0,000 \\
\hline
\end{tabular}

Coef B: Coeficientes Beta estimados usando modelos de regresión lineal simple y múltiple; IC 95\%: Intervalo de Confianza 95\%.

Fuente: elaboración propia. 


\section{Discusión}

En este estudio se evidenció que la mayoría de los estudiantes beneficiarios del PAE tenían alto nivel de percepción de satisfacción frente a las raciones alimentarias recibidas y más de la mitad de los participantes $(68 \%)$ consideró importante ser beneficiario del programa. Se confirmó que los estudiantes de menor edad estuvieron más satisfechos con los alimentos recibidos, tal como se observó en el PAE de Chile $^{12}$. Por su parte, los estudiantes de nivel socioeconómico bajo mostraron los mayores niveles de percepción de satisfacción con el programa, pues probablemente al recibir los alimentos sentían solventada, así fuera parcialmente, una de sus necesidades vitales. Esta relación de percepción positiva sobre acceso a alimentación y nivel socioeconómico bajo se ha identificado en otros estudios que sugieren que los sistemas psicológicos y fisiológicos que regulan el apetito también pueden ser sensibles a los sentimientos subjetivos de privación de recursos críticos no alimentarios ${ }^{18,19}$.

En el grupo de estudiantes pertenecientes a niveles socio económico medios, $25 \%$ en este estudio, un poco más de la mitad botaba los alimentos a la basura, hecho que también se presentó en el contexto de otros programas de alimentación escolar ${ }^{20,21}$ y que contribuye a profundizar la problemática relacionada con pérdida y desperdicios de alimentos en Colombia ${ }^{22}$.

$\mathrm{Al}$ analizar por grupos de edad la práctica de botar a la basura los alimentos no consumidos, se observó que fue mayor en los escolares de 5 a 8 años; este comportamiento podría corresponder a una tardía formación en el tema, tanto en la familia como en la escuela y en los medios de comunicación. Así, el desarrollo de procesos educativos y comunicativos adecuados y provocadores ${ }^{23,24}$ que orienten hacia la toma de conciencia temprana sobre el no desperdicio de alimentos es vital ${ }^{25}$. Estos hallazgos ameritan una revisión sobre el proceso de priorización y focalización del programa PAE de Bucaramanga, para ser más rigurosos en la selección de los estudiantes beneficiarios y lograr mejores resultados. En contraste con nuestros hallazgos, una revisión sistemática que incluyó 53 artículos sobre el Programa Nacional de Almuerzos Escolares en Estados Unidos encontró que los estudiantes mayores desperdiciaban más los alimentos ${ }^{26}$. Estas diferencias permiten recordar que, aunque el problema del desperdicio de alimentos es mundial y más intenso en países de ingreso medio y alto, sus causas son multidimensionales y deben ser estudiadas para un abordaje apropiado y efectivo si se desea incidir en el problema ${ }^{27}$. Cabe resaltar que en la percepción de satisfacción con los alimentos también inciden la cultura, los medios de comunicación, experiencias previas y creencias y actitudes, muy influenciadas por los pares ${ }^{28-31}$.

Sobre el tipo de ración, presentó mayor satisfacción en ambos grupos de edad la preparada en sitio, probablemente cercana en sabores a los del hogar. Sobre la ración industrializada, es importante monitorear los productos que se ofrecen pues la carga de aditivos químicos y azúcar que se usan en su fabricación pueden afectar la salud de los beneficiarios ${ }^{32}$. Diversos estudios han evidenciado que el consumo de estos por parte de la población infantil y adolescente, es inducida por la publicidad en televisión ${ }^{33}$ e incide en los programas de alimentación escolar, por tanto, el análisis de componentes nutricionales e ingredientes utilizados es esencial en el proceso de selección ${ }^{34,35}$.

Al evaluar percepción de satisfacción por componente, el sensorial fue el que obtuvo los mejores resultados, en sabor y olor para ambos grupos y en color de los alimentos en los más pequeños, resultado importante para la preparación de menús que estimulen el deseo de comer en este grupo etáreo ${ }^{36}$. Por su parte, el componente organizacional también tuvo alta percepción de satisfacción en el grupo de 5 a 8 años, mostrando cómo la satisfacción frente a la alimentación es más integral en los escolares pequeños ${ }^{37}$. Respecto al tiempo para consumo tranquilo de los alimentos, la mayoría de estudiantes indicaron ser presionados para comer, factor que se ha relacionado de manera negativa con la percepción de satisfacción pues reduce la posibilidad de socialización entre los escolares ${ }^{38}$. Sobre el componente social sobresale el manejo diferente en la variable Conversar con compañeros a la hora del refrigerio o almuerzo: los más pequeños no podían hacerlo, impidiéndose un momento importante de socialización y desarrollo del lenguaje ${ }^{39-41}$, en tanto que los más grandes sí, quienes además lo percibieron con alta satisfacción ${ }^{42}$.

En síntesis, la mayoría de los estudiantes estaban satisfechos y valoraban estar vinculados al programa PAE. El componente sensorial fue el que obtuvo los puntajes más altos en ambos grupos, el organizacional el mejor percibido por los escolares de 5 a 8 años y el social por los estudiantes de 9 a 17 años. Hay aspectos por mejorar especialmente en lo relacionado con la educación nutricional desde los primeros años de escolaridad para ayudar los niños a i) identificar el valor nutricional de los alimentos, ii) eliminar práctica 
de botarlos a la basura, iii) indagar sobre los alimentos industrializados que ven en la televisión, y iv) saber ejercer sus derechos y deberes como beneficiarios del programa. Es altamente recomendable fortalecerlos en sus habilidades psicosociales para la vida, las cuales les permitirán tener una mejor interacción con compañeros, docentes, personal del programa y lograr un mayor aprovechamiento del PAE y un mejoramiento permanente del mismo en su calidad a partir de los aportes de quienes reciben el servicio.

Es relevante remarcar que en el país hay baja producción científica sobre el tema, por tanto, es clave incrementar la investigación pues hace parte de las líneas de desarrollo humano y de progreso de Colombia y del Objetivo 2 de Desarrollo Sostenible 2030.

En este contexto, cobra relevancia toda evidencia sólida sobre el PAE en nuestro país, para profundizar el conocimiento sobre elementos esenciales que continúen mejorando y haciendo ajustes en la política pública pertinente, que permitan alcanzar en un mediano plazo mejores estándares de calidad y por ende, mayores beneficios para la población infantil y adolescente de estrato socioeconómico bajo en proceso de formación.

\section{Agradecimientos}

Las autoras agradecen al personal directivo en cada institución educativa su confianza y espíritu de colaboración para la recolección de los datos; a las Nutricionistas Dietistas del Instituto Proinapsa, Inés Bueno, Gloria Marconi y Elva Prieto quienes participaron en la coordinación y supervisión del trabajo de campo y al grupo de profesionales que recolectaron los datos para el estudio mediante la aplicación de las encuestas.

\section{Consideraciones éticas}

En la realización de esta investigación se tuvo en cuenta las consideraciones éticas planteadas en la Resolución 8430 de 1993 del Ministerio de Salud y Protección Social de Colombia. A los acudientes de los estudiantes se les informó sobre objetivos y beneficios de la investigación, recolección de datos mediante entrevista a los menores, participación voluntaria y libertad de retirar a sus hijos del estudio en cualquier momento; la participación de los menores, se formalizó con la firma del consentimiento informado por el acudiente. A los estudiantes mayores se les solicitó expresar su consentimiento en la reunión presencial luego de recibir información sobre el estudio, la recolección de datos en encuesta auto diligenciada, responder sus preguntas y remarcar la participación voluntaria. Este estudio corresponde a la categoría sin riesgo de acuerdo con el Artículo 11 de la mencionada Resolución.

\section{Conflicto de intereses}

Las autoras no tienen conflicto de intereses que declarar.

\section{Referencias}

1. Tembon A, Brooke L, Fernández E. School feeding: a tool for social inclusion. The World Bank. 2015.

2. Bundy DA, Drake LJ, Burbano C. School food, politics and child health. Public Health Nutr. 2013; 16(6): 1012-1019. doi:http://dx.doi.org/10.1017/S1368980012004661.

3. Cooperación Brasil - FAO. Fortalecimiento de programas de alimentación escolar en el marco de la iniciativa América Latina y Caribe sin hambre 2025. Proyecto GCP/RLA/180/BRA. 2013.

4. Programa Mundial de Alimentos - WFP. Nutrir el Futuro: Programas de Alimentación Escolar Sensibles a la Nutrición en América Latina y el Caribe. Un Estudio de 16 Países. 2017.

5. Ospina S, Gil I. Índices nacionales de satisfacción del consumidor. Una propuesta de revisión de la literatura. Cuad Admin. 2011; 24(43): 35-57

6. Schnettler B, Miranda H, Orellana L, Sepúlveda J, Sepúlveda J, Mora M, et al. Variables que afectan la satisfacción con la alimentación según nivel socioeconómico: un estudio exploratorio en el sur de Chile. Rev Chil Nutr. 2014; 41(2): 149-155. doi: http://dx.doi.org/10.4067/S0717-75182014000200004.

7. Rubio G, Rodríguez M S, Uribe ME. Análisis de la percepción de los clientes respecto a la calidad del servicio recibido por parte del personal que atiende en las grandes superficies de la ciudad de Ibagué. Dimens Empres 2012; 10(2): 21-31.

8. Colombia - Ministerio de Educación Nacional. Lineamientos técnicos y administrativos, los estándares y las condiciones mínimas del Programa de alimentación escolar - PAE. Resolución 16432; 2015.

9. World Food Program - WFP. Colombia Annual Country Report - Country Strategic Plan April 2017- December 2021.

10. Martínez LI. Los programas de alimentación escolar en América Latina. Arch Latinoam Nutr. 2015; 65(Suppl 1): 156.

11. Colombia - Secretaria de Educación Departamental Vaupés. Programa de restaurante escolar. Grado de satisfacción del programa de restaurante escolar; 2014. 
12. Junta Nacional de Auxilio Escolar y Becas JUNAEB. ClioDinámica Asesorías, Consultoría e Ingeniería Limitada. Estudio evaluación de la satisfacción de los usuarios del programa de alimentación escolar PAE - JUNAEB. Chile; 2017.

13. Nessier MC, Monsalvo A, Picech V, Pacheco $\mathrm{J}$, Palma V, Martínez MS, et al. Percepción de usuarios del programa de recuperación del niño en riesgo nutricional "Nutrir más" del área urbana de la ciudad de Santa Fe, Argentina. Rev Chil Nutr. 2010; 37(1): 51-59. doi: http://dx.doi.org/10.4067/S0717-75182010000100005.

14. Sanabria MC, Frutos D, Preda J, Gónzalez L, Cornelli P. Adecuación y aceptación de almuerzos escolares en dos escuelas públicas de Asunción. Pediatr (Asunción). 2017; 44(2): 126-135. doi: http://dx.doi.org/10.18004/ped.2017.agosto.126-135.

15. Jisook J, Youngmee L, Yu-jin O. Comparison if student's satisfaction on school food service environment by eating place and gender. Nutr Res Pract. 2009; 3(4): 295-299. doi: http://dx.doi.org/10.4162 / nrp.2009.3.4.295.

16. Smith S, Cunningham-Sabo L, Auld G. Satisfaction of middle school lunch program participants and non-participants with the school lunch experience. J Child Nutr Manag. 2015; 39(2).

17. Franco SM. Aportes de la sociología al estudio de la alimentación familiar. Luna Azul. 2010; (31): 139-155.

18. Cheon BK, Hong Y. Mere experience of low subjective socioeconomic status stimulates appetite and food intake. Proc Natl Acad Sci USA. 2017; 114(1): 72-77. doi: https://doi.org/10.1073/pnas.1607330114.

19. Vedovato GM, Surkan PJ, Jones-Smith J, Steeves EA, Han E, Trude AC, et al. Food insecurity, overweight and obesity among low-income African-American families in Baltimore City: associations with food-related perceptions. Public Health Nutr. 2016; 19(8): 1405-1416. doi: http://dx.doi.org/10.1017 / S1368980015002888.

20. Arévalo Porras O. Análisis del desperdicio de alimentos en el almuerzo escolar del Colegio Distrital Ciudadela Educativa, una mirada desde las dimensiones de la Seguridad Alimentaria y Nutricional. Trabajo de Grado para obtener título de Magister en Seguridad Alimentaria y Nutricional. Universidad Nacional de Colombia. Bogota, Colombia. 2016.

21. Pikelaizen C, Neumann Spinelli MG. Avaliação do desperdício de alimentos na distribuição do almoço servido para estudantes de um colégio privado em São Paulo, SP. Rev Univap. 2013; 19(33): 5-12. doi: http://dx.doi.org/10.18066/revunivap.v19i33.111.

22. Colombia - Departamento Nacional de Planeación Dirección de Seguimiento y Evaluación de Políticas Públicas. Pérdida y desperdicios de alimentos en Colombia. 2016.

23. Calero S. La comida y la comensalidad como escenarios comunicativos. Diálogos Comunic. 2014; 89: 1-24.

24. Trescastro EM, Trescastro S. La educación en alimentación y nutrición en el medio escolar: el ejemplo del Programa EDALNU. Rev Esp Nutr Hum Diet. 2013. 17(2): e84-e90.

25. Organización de las Naciones Unidas para la Alimentación y la Agricultura. El futuro de la alimentación y la agricultura: tendencias y desafíos. FAO, 2017.

26. Byker C, Banna J, Serrano E. Food waste in the national school lunch program 1978-2015: a systematic review. Acad Nutr Diet. 2017; 117(11): 1792-1807. doi: http://dx.doi.org/10.1016/j.jand.2017.06.008.

27. Basso N, Brkic M, Moreno C, Pouiller P, Romero A. Valoremos los alimentos, evitemos pérdidas $\mathrm{y}$ desperdicios. Diaeta. 2016; 34(155): 25-32.

28. Atik D, Ozdamar Z. Children's perception of food and healthy eating: Dynamics behind their food preferences. Int J Consum Stud. 2013; (37): 59-65.

29. Román V, Quintana M. Nivel de influencia de los medios de comunicación sobre la alimentación saludable en adolescentes de colegios públicos de un distrito de Lima. An Fac Med. 2010; 71(3):185189. doi: https://doi.org/10.15381/anales. v71i3.93.

30. Díaz-Beltrán M. Factores influyentes en el comportamiento alimentario infantil. Rev Fac Med. 2014; 62(2): 237-245. doi: https://doi.org/10.15446/revfacmed.v62n2.45414.

31. Aranceta Bartrina J. Influencia de los medios de comunicación en la elección de alimentos y en los hábitos de consumo alimentario. Tesis doctoral. Universidad del País Vasco; 2015.

32. Bejarano JJ, Suárez LM. Algunos peligros químicos y nutricionales del consumo de los alimentos de venta en espacios públicos. Rev Univ Ind Santander Salud. 2015; 47(3): 349-360. doi: http://dx.doi.org/10.18273/revsal.v47n3-201501.

33. Sevillano ML, Sotomayor A. Publicidad y consumo de alimentos en estudiantes de Huánuco, Perú. Comunicar. 2012; 20(39): 177-184. doi: https://doi.org/10.3916/C39-2012-03-08.

34. Freire WB, Waters WF, Rivas-Mariño G. Semáforo nutricional de alimentos procesados: estudio cualitativo sobre conocimientos, comprensión, actitudes y prácticas en el Ecuador. Rev Peru 
Med Exp Salud Publica. 2017; 34(1): 11-18. doi: http://dx.doi.org/10.17843/rpmesp.2017.341.2762.

35. Torres F, Lutz B. Papel de la industria alimentaria y de la sociedad civil en los comedores comunitarios de SINHAMBRE. Casos de la Montaña y centro de Guerrero. Espiral. 2016; 23(67): 239-277.

36. Paquet C, de Montigny L, Labban A, Buckeridge $\mathrm{D}$, Ma Y, Arora $\mathrm{N}$, et al. The moderating role of food cue sensitivity in the behavioral response of children to their neighborhood food environment: a cross-sectional study. Int J Behav Nutr Phys Act. 2017; 14:86. doi: https://doi.org/10.1186/s12966-017-0540-9.

37. Maietta OW, Gorgitano MT. School meals and pupil satisfaction. Evidence from Italian primary schools. Food Policy. 2016; 62: 41-55.

38. Fraire M, Longobardi C. Niños a la hora de la comida: un análisis de interacción conversacional. Int J Develop Educ Psy. 2008; 1: 261-270.

39. Corredor J, Romero C. Apuntes lingüísticos, discursivos y pedagógicos sobre la conversación constructiva. Cuad Ling Hisp. 2010; 15: 93-108.

40. Serrano J. Desarrollo de la teoría de la mente, lenguaje y funciones ejecutivas en niños de 4 a 12 años. Universidad de Girona - Tesis doctoral; 2012.

41. Amaya M, Osorio O. The feeding of adolescents: the place and the company determine the alimentary practices. Aquichan. 2011; 11: 199-216.

42. Kwon SH, Cha M, Kim YK. High school students' satisfaction with foodservice quality is affected by foodservice management type. Int J Food Sci Nutr. 2005; 10: 372-377. 